\title{
CURVATURE, DIAMETER AND BOUNDED BETTI NUMBERS
}

\author{
Zhongmin Shen AND JyH-YANG Wu
}

Feb. 1999

\section{INTRODUCTION}

One of the fundamental problems in Riemannian geometry is to understand the relation between the topology and geometry of a Riemannian manifold.

Every closed manifold $M$ admits a Riemannian metric $g$ with the following curvature bound

$$
K \geq-1
$$

Thus the curvature bound in (1.1) alone does not have any implication for the topological structure of the manifold. With this normalized metric, the topology depends on the "size" of the manifold. The diameter is one of the global geometric quantities to measure the manifold. Assume that

$$
\text { Diam } \leq D
$$

It was proved by M. Gromov [G1] that for any Riemannian $n$-manifold $(M, g)$ satisfying (1.1) and (1.2), the total Betti number (with respect to any field) is bounded, namely,

$$
\sum_{i=0}^{n} \beta^{i}(M) \leq C(n, D) .
$$

In this paper, we are interested in a much weaker curvature bound

$$
\operatorname{Ric} \geq-(n-1)
$$

First of all, according to Sha-Yang's examples [SY], the Gromov Betti Number Theorem is not true for Riemannian $n$-manifolds satisfying (1.2) and (1.3). Nevertheless, the first Betti number is still bounded, i.e.,

$$
\beta^{1}(M) \leq C(n, D)
$$

This is due to M. Gromov [GLP] and T. Gallot [GT].

Besides the ordinary Betti number, what topological invariants are still controlled by the curvature bounds (1.1) or (1.3) ? To answer this question, we consider the

J.-Y. Wu is partially supported by a Taiwan NSC grant. 
bounded cohomology groups $\hat{\mathrm{H}}^{*}(M)$. The natural inclusion $I: \hat{C}^{*}(M) \rightarrow C^{*}(M)$ induces a homomorphism $\iota: \hat{\mathrm{H}}^{*}(M) \rightarrow \mathrm{H}^{*}(M)$ with image $\tilde{\mathrm{H}}^{*}(M):=\iota\left[\hat{\mathrm{H}}^{*}(M)\right]$ (see Section 2 below for details). Put

$$
\begin{aligned}
& \tilde{\beta}^{i}(M):=\operatorname{dim} \tilde{\mathrm{H}}^{i}(M), \\
& \hat{\beta}^{i}(M):=\operatorname{dim} \hat{\mathrm{H}}^{i}(M) .
\end{aligned}
$$

Clearly,

$$
\tilde{\beta}^{*}(M) \leq \beta^{*}(M), \quad \tilde{\beta}^{*}(M) \leq \hat{\beta}^{*}(M) .
$$

But there is no direct relationship between $\beta^{*}(M)$ and $\hat{\beta}^{*}(M)$. Both $\tilde{\beta}^{i}(M)$ and $\hat{\beta}^{i}(M)$ are called the $i$ th bounded Betti numbers of $M$. Below are some important examples: 0 ;

(1)(Gromov) For any closed manifold $M, \hat{\mathrm{H}}^{1}(M)=0$, hence $\tilde{\beta}^{1}(M)=\hat{\beta}^{1}(M)=$

(2)(Thurston) For any closed manifold of negative curvature, $\tilde{\mathrm{H}}^{k}(M)=\mathrm{H}^{k}(M)$ for all $k \geq 2$, hence $\tilde{\beta}^{k}(M)=\beta^{k}(M)$ for $k \geq 2$;

(3) (Trauber) If $\pi_{1}(M)$ is amenable, then $\hat{\mathrm{H}}^{*}(M)=0$, hence $\tilde{\beta}^{*}(M)=\hat{\beta}^{*}(M)=$ 0 .

The bounded Betti numbers behave quite differently from the ordinary Betti numbers. In particular, the Poincaré duality for $\beta^{*}(M)$ does not hold for $\tilde{\beta}^{*}(M)$ and $\hat{\beta}^{*}(M)$. By [Gr3], we know that the bounded Betti numbers $\hat{\beta}^{*}(M)$ are completely determined by $\pi_{1}(M)$. However, the bounded Betti numbers $\tilde{\beta}^{*}(M)$ depend not only on $\pi_{1}(M)$, but also on the higher dimensional topological structure of $M$, when $\pi_{1}(M)$ is not amenable.

In this paper, we shall prove the following

Theorem A. There is a constant $C(n, D)$ depending only on $n, D$ such that for closed Riemannian $n$-manifold $(M, g)$ satisfying Ric $\geq-(n-1)$ and Diam $\leq D$, the total bounded Betti number is bounded

$$
\sum_{i=0}^{n} \tilde{\beta}^{i}(M) \leq C(n, D) .
$$

It is proved by Gromov that there is a small number $\epsilon(n)>0$ such that if a closed oriented $n$-manifold $M$ satisfies (1.3) and

$$
\sup _{p \in M} \operatorname{vol}(B(p, 1))<\epsilon(n),
$$

then there is a map $f$ from $M$ into an $(n-1)$-dimensional polyhedron $P^{n-1}$ such that the pre-image of any star neighborhood is contained in an amenable open subset. Then he concludes that $\|M\|=0$, which is equivalent to that $\tilde{\beta}^{n}(M)=0$. 
Theorem B. Let $X$ be a compact metric space. There is a small constant $\epsilon=$ $\epsilon(n, X)>0$ such that for any closed $n$-manifold $M$ satisfying Ric $\geq-(n-1)$, if $d_{G H}(M, X)<\epsilon$, then $\tilde{\beta}^{i}(M)=0$ for $i>\operatorname{dim} X$.

Under a stronger curvature bound, the bounded cohomology groups $\hat{\mathrm{H}}^{*}(M)$ of $M$ are controlled too. More precisely,

Theorem C. There are only finitely many isometric isomorphism types of bounded cohomology groups $\left(\hat{H}^{*}(M),\|\cdot\|_{\infty}\right)$ among closed Riemannian n-manifolds satisfying $K \geq-1$ and Diam $\leq D$.

From Theorem $\mathrm{C}$, we conclude that there is a constant $C(n, D)$ such that if a closed $n$-manifold satisfying (1.1) and (1.2), then

$$
\hat{\beta}^{*}(M) \leq C(n, D)
$$

provided that $\hat{\beta}^{*}(M)<\infty$. However, there are closed manifolds $M$ with $\hat{\beta}^{*}(M)=$ $\infty$. Take $M=S^{1} \times S^{4} \# S^{1} \times S^{4}$. The fundamental group $\pi_{1}(M)=Z * Z$. Thus the second bounded cohomology group $\hat{\mathrm{H}}^{2}(M)$ is not finitely generated. This example is given to us by F. Fang.

\section{Preliminaries}

In this section, we shall summarize some of Gromov's results [G3] which will be needed in our proof.

Let $M$ be a connected topological space. Denote by $\Sigma$ the set of all singular simplices $\sigma: \Delta \rightarrow M$. The standard pseudo- $L^{\infty}$ norm on $C^{*}(M)$ is defined by

$$
\|c\|_{\infty}:=\sup _{\sigma \in \Sigma}|c(\sigma)|
$$

Consider the subcomplex $\hat{C}^{*}(M)$ of bounded singular cochains of $M$. The homology groups $\hat{\mathrm{H}}^{*}(M)$ of $\hat{C}^{*}(M)$ is called the bounded cohomology of $M$. Let $\|\cdot\|_{\infty}^{b}$ denote the induced norm on $\hat{\mathrm{H}}^{*}(M)$. Then $\left(\hat{\mathrm{H}}^{*}(M),\|\cdot\|_{\infty}^{b}\right)$ becomes a normed space. The natural inclusion $I: \hat{C}^{*}(M) \rightarrow C^{*}(M)$ induces a homomorphism

$$
\iota: \hat{\mathrm{H}}^{*}(M) \rightarrow \mathrm{H}^{*}(M)
$$

Put

$$
\tilde{\mathrm{H}}^{*}(M):=\iota\left[\hat{\mathrm{H}}^{*}(M)\right]
$$

$\tilde{\mathrm{H}}^{*}(M)$ is called the bounded part of $\mathrm{H}^{*}(M)$ (see $\left.[\mathrm{Br}]\right)$. Cohomology classes in $\tilde{\mathrm{H}}^{*}(M)$ are called bounded classes of $\mathrm{H}^{*}(M)$. Put

$$
\|\alpha\|_{\infty}:=\inf _{\beta \in I^{-1}(\alpha)}\|\beta\|_{\infty}^{b}
$$


The most important fact in the bounded cohomology theory is that the normed space $\left(\hat{\mathrm{H}}^{*}(M),\|\cdot\|_{\infty}^{b}\right)$ actually depends only on the fundamental group $\pi_{1}(M)$ ([G3]) and $\hat{\mathrm{H}}^{*}(M)=0$ for connected closed manifolds with $\pi_{1}(M)$ amenable.

Below, we shall sketch Gromov's ideas to prove the above fact. One is referred to $[\mathrm{Br}][\mathrm{I}]$ for different arguments. Although Gromov's theory is for general topological spaces, we shall focus on closed manifolds.

First, Gromov introduced a notion of multipcomplex. A (simplicial) multicomplex is defined as a set $K$ divided into the union of closed affine simplices $\Delta_{\sigma} \subset K$, $\sigma \in I$ such that the intersection of any two simplices $\Delta_{\sigma} \cap \Delta_{\tau}$ is a subcomplex in both $\Delta_{\sigma}$ and $\Delta_{\tau}$. The set $K$ with the weakest topology which agrees with the decomposition $K=\cup_{\sigma \in I} \Delta_{\sigma}$ is denoted by $|K|$. The union of all $i$-dimensional simplices in $K$ is called the $i$-skeleton of $K$, denoted by $K^{i} \subset K$.

Let $M$ be an $n$-dimensional closed manifold. Denote by $\Sigma$ the set of all singular simplicies $\sigma: \Delta^{i} \rightarrow M, i=0,1,2, \cdots$, which are injective on the vertices of the standard (oriented) $i$-simplex $\Delta^{i}$. Take one copy of $\Delta^{i}$ for each $\sigma$, denoted by $\Delta_{\sigma}^{i}$, and put $K:=\cup_{\sigma \in \Sigma} \Delta_{\sigma}^{i}$. This union has a natural structure of a multicomplex such that the canonical map: $S:|K| \rightarrow M$ defined by $\left.S\right|_{\Delta_{\sigma}^{i}}:=\sigma: \Delta_{\sigma}^{i} \rightarrow M$, is continuous. Gromov proves that $S$ is a weak homotopy equivalence. The multicomlex $K$ is large and complete in the sense that every component of $K$ has infinitely many vertices, and every continuous map $f: \Delta^{i} \rightarrow K$ is homotopic, relative to $\partial \Delta^{i}$, to a simplicial embedding $g: \Delta^{i} \rightarrow K$, provided $\left.f\right|_{\partial \Delta^{i}}: \partial \Delta^{i} \rightarrow K$ is a simplicial embedding.

For the multicomplex $K$ constructed above, there is another natural notion of bounded cohomology $\hat{\mathrm{H}}_{a}^{*}(K)$. Let $\hat{C}_{a}^{*}(K)$ denote the complex of bounded antisymmetric real cochains $c$, that is, $c\left(\Delta_{\sigma}^{i}\right)=-c\left(\Delta_{\sigma \circ \delta}^{i}\right)$ for any orientation-preserving affine isomorphism $\delta: \Delta^{i} \rightarrow \Delta^{i}$. Then $\hat{\mathrm{H}}_{a}^{*}(K)$ is defined to be the homology group of $\hat{C}_{a}^{*}(K)$ with the natural pseudo-norm $L_{\infty}$. Define a homomorphism $h: \hat{C}^{i}(M) \rightarrow \hat{C}_{a}^{i}(K)$ by

$$
h\left(\Delta_{\sigma}^{i}\right):=\frac{1}{(i+1) !} \sum_{\delta}[\delta] c(\sigma \circ \delta) .
$$

Gromov asserts that $h$ induces an isometric isomorphism

$$
h^{*}: \hat{\mathrm{H}}^{i}(M) \rightarrow \hat{\mathrm{H}}_{a}^{i}(K) \text {. }
$$

In order to prove the fact that $\hat{\mathrm{H}}^{*}(M)$ actually depend only on $\pi_{1}(M)$, Gromov introduces a large and complete subcomplex $i: \tilde{K} \hookrightarrow K$ with the following properties:

(i) each continuous map of a simplex $\Delta^{i}$ into $\tilde{K}$, whose restriction to the boundary is a simplicial embedding, is homotopic relative to the boundary $\partial \Delta^{i}$ to at most one simplicial embedding $\Delta^{i} \rightarrow K$.

(ii) the natural inclusion $i: \tilde{K} \hookrightarrow K$ is a homotopy equivalence. Hence it induces an isometric isomorphism 
A subcomplex $\tilde{K}$ with these properties exists and is uniquely determined, up to an simplicial isomorphism, by the homotopy type of $K . \tilde{K}$ is called a minimal model of $K$.

Fix a minimal model $\tilde{K}$ of $K$. Let $\Gamma_{1}=\Gamma_{1}(\tilde{K})$ denote the group of simplicial automorphisms of $\tilde{K}$ which are homotopic to the identity and keeps the 1-skelton of $\tilde{K}$ fixed. Then $\tilde{K}_{1}:=\tilde{K} / \Gamma_{1}$ is a $K(\pi, 1)$ multicomplex with $\pi=\pi_{1}(\tilde{K})=\pi_{1}(M)$ and the projection $p: \tilde{K} \rightarrow \tilde{K}_{1}$ induces an isomorphism between fundamental groups. In particular, the projection $p: \tilde{K} \rightarrow \tilde{K}_{1}$ induces an isometric isomorphism

$$
p^{*}: \hat{\mathrm{H}}_{a}^{*}\left(\tilde{K}_{1}\right) \rightarrow \hat{\mathrm{H}}_{a}^{*}(\tilde{K}) .
$$

In virtue of (2.4)-(2.6), one can conclude that

$$
\Phi:=p^{*-1} \circ i^{*} \circ h^{*}: \hat{\mathrm{H}}^{*}(M) \rightarrow \hat{\mathrm{H}}_{a}^{*}\left(\tilde{K}_{1}\right)
$$

is an isometric isomorphism. Thus Gromov concludes that the normed cohomology groups $\hat{\mathrm{H}}^{*}(M)$ depend only on $\pi_{1}(M)$.

Let $\tilde{\Gamma}:=\oplus_{x \in \tilde{K}_{1}^{0}} \pi_{1}\left(\tilde{K}_{1}, x\right)$. The group $\tilde{\Gamma}$ acts on $\tilde{K}_{1}$ in a natural way. Assume that $\pi_{1}(M)$ is amenable, then $\tilde{\Gamma}$ is amenable. The standard averaging process leads to the following remarkable conclusion: $\hat{\mathrm{H}}^{*}(M)=\hat{\mathrm{H}}_{a}^{*}(\tilde{K})=0$. By a similar argument, one can show that the amenable normal subgroups of $\pi_{1}(M)$ make no contributions to the bounded cohomology $\hat{\mathrm{H}}^{*}(M)$. More precisely, we have the following

Lemma 2.1. Let $\Gamma \subset \pi_{1}(M)$ be a normal amenable subgroup. Then $\Gamma$ induces an action $\tilde{\Gamma}$ on $\tilde{K}_{1}$ such that $\tilde{K}_{1} / \tilde{\Gamma}$ is a multicomplex of $K(\pi, 1)$ type with $\pi=\pi_{1}(M) / \Gamma$ and $\hat{H}^{*}(M)$ is isometric isomorphic to $\hat{H}_{a}^{*}\left(\tilde{K}_{1} / \tilde{\Gamma}\right)$.

Consider a class $\mathcal{M}$ of certain closed $n$-manifolds. Let

$$
\begin{aligned}
& \mathcal{M}_{\pi}:=\left\{\pi_{1}(M), M \in \mathcal{M}\right\} / \sim \\
& \hat{\mathcal{M}}^{i}:=\left\{\left(\hat{\mathrm{H}}^{i}(M),\|\cdot\|_{\infty}^{b}\right), M \in \mathcal{M}\right\}
\end{aligned}
$$

where $\pi_{1}(M) \sim \pi_{1}\left(M^{\prime}\right)$ if and only if there are normal amenable subgroups $N \triangleleft$ $\pi_{1}(M)$ and $N^{\prime} \triangleleft \pi_{1}\left(M^{\prime}\right)$ such that $\pi_{1}(M) / N \approx \pi_{1}\left(M^{\prime}\right) / N^{\prime}$. Suppose that there are only finitely many isomorphism types of $\pi_{1}(M)$ in $\mathcal{M}_{\pi}$. By Lemma 2.1, one can conlude that there are only finitely isometric isomorphism types of normed spaces $\left(\hat{\mathrm{H}}^{*}(M), \cdot \|_{\infty}^{b}\right)$ in $\hat{\mathcal{M}}^{i}$ for each $i$.

We now consider the bounded part $\tilde{\mathrm{H}}^{*}(M)$ of $\mathrm{H}^{*}(M)$. Althought $\tilde{\mathrm{H}}^{*}(M)$ is the image of $\hat{\mathrm{H}}^{*}(M)$, it is not clear how does it depend on the fundamental group. In certain cases, the bounded cohomology group is very large, while the bounded part is trivial. Look at a closed integral homology 3 -spheres $M$ with a hyperbolic metric. In this case, $\tilde{\mathrm{H}}^{*}(M)=\mathrm{H}^{*}(M)=0$, but $\hat{\mathrm{H}}^{2}(M) \neq 0$.

It is natural to consider the case when a compact manifold $M$ is covered by a 
any $x \in U$, the image of the inclusion $i_{*}: \pi_{1}(U, x) \rightarrow \pi_{1}(M, x)$ is an amenable subgroup. One expects that $\tilde{\mathrm{H}}^{*}(M)$ might be controlled by an amenable covering of the manifold. Based on Gromov's bounded cohomology theory, N. V. Ivanov [I] has made an important observation. He proved an analog of Leray's theorem on amenable coverings.

Lemma 2.2. ([I]) Let $M$ be an $n$-dimrensional manifold, $\mathcal{U}$ be an amenable covering of $M, N$ be the nerve of this covering, and $|N|$ be the geometric realization of the nerve. Then the canonical map $\iota: \hat{H}^{*}(M) \rightarrow H^{*}(M)$ factors through the map $\phi: H^{*}(|N|) \rightarrow H^{*}(M)$. In other words, there is a homomorphism $\psi: \hat{H}^{*}(M) \rightarrow$ $H^{*}(|N|)$ such that $\iota=\phi \circ \psi$.

\section{Proofs of Theorems A And B}

Before we start to prove Theorem A, we recall a generalized version of Margulis' lemma which is due to Fukaya and Yamaguchi ([FY1]) in the sectional curvature case and then extended by Cheeger and Colding ([CC1]) to the Ricci curvature case.

Margulis' Lemma. Given $n$ and $k$, there exists a positive number $\epsilon(n)$ depending only on $n$ and $k$ such that if $M$ is a complete Riemannian n-manifold with (1.3), then the image of the inclusion map $i_{*}: \pi_{1}(B(p, r), p) \rightarrow \pi_{1}(M)$ is almost nilpotent for any point $p \in M$ when $r \leq \epsilon(n)$.

Proof of Theorem $A$ : Let $(M, g)$ be a closed Riemannian $n$-manifold satisfying $(1.2)$ and (1.3). Let $r=\epsilon(n)$ be the number in Margulis's Lemma. Take a maximal set of disjoint $r / 2$-balls $B\left(p_{i}, r / 2\right), i=1, \cdots, m$. Then $\mathcal{U}:=\left\{B\left(p_{i}, r\right)\right\}_{i=1}^{m}$ cover $M$. Assume that $B\left(p_{i_{o}}, r / 2\right)$ has the smallest volume among $B\left(p_{i}, r / 2\right)$. By the Bishop-Gromov volume comparison, we obtain

$$
m \leq \frac{\operatorname{vol}(M)}{\operatorname{vol}\left(B\left(p_{i_{o}}, r / 2\right)\right)} \leq \frac{\int_{0}^{D} \sinh ^{n-1}(t) d t}{\int_{0}^{r / 2} \sinh ^{n-1}(t) d t}=C(n, D) .
$$

Let $N$ be the nerve of this covering $\mathcal{U}$ and $|N|$ be the geometric realization of the nerve. Since the number of the simplices in $N$ is bounded by $C(n, D)$, there is constant $C^{\prime}(n, D)$ depending on $C(n, D)$ such that

$$
\operatorname{dim} \mathrm{H}^{*}(|N|) \leq C^{\prime}(n, D) .
$$

Note that each ball $B\left(x_{i}, r\right)$ in $\mathcal{U}$ is amenable. By Lemma 2.2, we conclude that

$$
\tilde{\beta}^{*}(M) \leq \operatorname{dim} \mathrm{H}^{*}(|N|) \leq C^{\prime}(n, D) .
$$

This proves Theorem A.

The discussions above also suggest the following

Problem. Are there finitely many isometric isomorphism types of $\left(\tilde{H}^{*}(M),\|\cdot\|_{\infty}\right)$ among closed $n$-manifolds $(M, g)$ satisfying (1.2) and (1.3)?

Since there are infinitely many normed spaces in each dimension, Theorem A 
Proof of Theorem B. Let $X$ be as in Theorem B. Since $X$ is compact, we can take a finite open covering $\left\{W_{j}\right\}$ of $X$ with mesh $<\epsilon(n) / 8$ and order $\leq \operatorname{dim} X+1$, where $\epsilon(n)$ is given by the Margulis' Lemma. That is, $\operatorname{Diam}\left(W_{j}\right)<\epsilon(n) / 4$ for all $j$ and every point $x$ is contained no more than $\operatorname{dim} X+1$ subsets $W_{j}$.

Claim 1. There is a positive number $\delta$ such that every geodesic ball $B(x, \delta)$ in $X$ is contained in some $W_{j}$.

Indeed, if this is not true, we can find a sequence of points $x_{i}$ in $X$ and positive numbers $\delta_{i} \rightarrow 0$ such that the geodesic ball $B\left(x_{i}, \delta_{i}\right)$ is not totally contained in any $W_{j}$ for all $i$. Since $X$ is compact, we can find, by taking a subsequence if necessary, a limit point $x$ of $x_{i}$ in $X$. But now, the point $x$ must be in some $W_{j}$ and hence $W_{j}$ contains a geodesic ball $B(x, r)$ for some positive raduis $r>0$. Then the triangle inequality implies that the geodesic ball $B\left(x_{i}, \delta_{i}\right)$ is contained in $W_{j}$ for large $i$. This gives a contradiction and Claim 1 holds.

Next we consider the closed complement $F_{j}$ of $W_{j}$ in $X, F_{j}:=X-W_{j}$, and set

$$
E_{j}:=\left\{x \in X \mid d\left(x, F_{j}\right) \geq \frac{\delta}{2}\right\}
$$

The set $E_{j}$ is closed and the triangle inequality implies that $\left\{E_{j}\right\}$ is a closed covering of $X$ due to our choice of $\delta$.

We take the positive number $\delta(X)$ to be the minimum of $\delta / 8$ and $\epsilon(n) / 8$.

Assuming the Gromov-Hausdorff distance between $M$ and $X$ is less than $\delta(X)$, we can find an admissible metric $d$ on the disjoint union $M \amalg X$ such that the classical Hausdorff distance of $M$ and $X$ in $M \amalg X$ is less than $\delta(X)$. Then we define an open covering $\mathcal{U}=\left\{U_{j}\right\}$ of $M$ by setting

$$
U_{j}:=\left\{p \in M: d_{M \amalg X}\left(p, E_{j}\right)<2 \delta(X)\right\}
$$

The triangle inequality then gives that $\mathcal{U}$ has mesh less than $\epsilon(n)$ and it covers $M$.

Claim 2. The order of this open covering $\mathcal{U}$ of $M$ is at most $\operatorname{dim} X+1$.

Indeed, if there is a point $p$ in $m=(\operatorname{dim} X+2)$ different open sets in $\mathcal{U}$, say, $U_{j}, j=1, \cdots, m$, then we can find a point $x \in X$ with $d_{M \amalg X}(p, x)<\delta(X)$ and the triangle inequality gives $d\left(x, E_{j}\right)<3 \delta(X)$. Hence, one has $d\left(p, F_{j}\right) \geq \frac{\delta}{2}-\frac{3 \delta}{8}=\frac{\delta}{8}>$ 0 and thus $p \in W_{j}$ for $j=1,2, \ldots, m$. This contradicts to the order of the covering $\left\{W_{j}\right\}$ since $m=\operatorname{dim} X+2$ and Claim 2 follows.

Therefore, we obtain an amenable open covering $\mathcal{U}$ of $M$ when $d_{H}(M, X)<\delta(X)$. Let $N$ be the nerve of this covering $\mathcal{U}$ and $|N|$ be the geometric realization of the nerve. By our construction,

$$
\operatorname{dim}|N| \leq \operatorname{dim} X
$$

Thus

$$
\mathrm{H}^{i}(|N|)=0, \quad i>\operatorname{dim} X
$$




\section{Proof of Theorem C}

In this section we shall prove Theorem C. First, we recall the notion about the equivariant Hausdorff distance from [FY1]. Let $\mathcal{M}_{\text {met }}$ denote the set of all isometry classes of pointed inner metric spaces $(X, p)$ such that for each $r$ the ball $B(p, r)$ is relatively compact in $X$. Let $\mathcal{M}_{e q}$ be the set of triples $(X, G, p)$ where $(X, p)$ is in $\mathcal{M}_{\text {met }}$ and $G$ is a closed group of isometries of $X$. For $r>0$, put

$$
G(r)=\{g \in G \mid d(g p, p)<r\} .
$$

Definition 4.1. Let $(X, G, x),(Y, H, y)$ be in $\mathcal{M}_{e q}$. An $\epsilon$-equivariant pointed Hausdorff approximation stands for a triple $(f, \phi, \psi)$ of maps $f: B\left(x, \frac{1}{\epsilon}\right) \rightarrow Y, \phi$ : $G\left(\frac{1}{\epsilon}\right) \rightarrow H\left(\frac{1}{\epsilon}\right)$ and $\psi: H\left(\frac{1}{\epsilon}\right) \rightarrow G\left(\frac{1}{\epsilon}\right)$ such that

(1) $f(x)=y$,

(2) the $\epsilon$-neighborhood of $f\left(B\left(x, \frac{1}{\epsilon}\right)\right)$ contains $B\left(y, \frac{1}{\epsilon}\right)$,

(3) if $p, q \in B\left(x, \frac{1}{\epsilon}\right)$, then $|d(f(p), f(q))-d(p, q)|<\epsilon$,

(4) if $p \in B\left(x, \frac{1}{\epsilon}\right), g \in G\left(\frac{1}{\epsilon}\right), g p \in B\left(x, \frac{1}{\epsilon}\right)$, then $d(f(g p), \phi(g)(f(p)))<\epsilon$,

(5) if $p \in B\left(x, \frac{1}{\epsilon}\right), h \in H\left(\frac{1}{\epsilon}\right), \psi(h)(p) \in B\left(x, \frac{1}{\epsilon}\right)$, then $d(f(\psi(h)(p)), h(f(p)))<$ $\epsilon$.

We remark that it is required neither that $f$ is continuous nor that $\phi, \psi$ are homomorphisms. The equivariant pointed Hausdorff distance $d_{e H}((X, G, x),(Y, H, y))$ is defined to be the infimum of the positive numbers $\epsilon$ such that there exist $\epsilon$ equivariant Hausdorff approximations from $(X, G, x)$ to $(Y, H, y)$ and from $(Y, H, y)$ to $(X, G, x)$. By $d_{H}$ we denote the pointed Hausdorff distance, which is the case when the groups are trivial. The notion

$$
\lim _{i \rightarrow \infty}\left(X_{i}, G_{i}, x_{i}\right)=(Y, G, y)
$$

means

$$
\lim _{i \rightarrow \infty} d_{e H}\left(\left(X_{i}, G_{i}, x_{i}\right),(Y, H, y)\right)=0 .
$$

Now we proceed to prove Theorem $\mathrm{C}$ by the method of absurity as in [W2]. Suppose Theorem $\mathrm{C}$ were false. Then, there exists a sequence of Riemannian $n$ manifolds $M_{j}$ satisfying $K \geq-1$ and Diam $\leq D$ such that all of their bounded cohomology $\hat{\mathrm{H}}^{*}\left(M_{j}\right)$ are different.

Choose a base point $x_{j}$ in $M_{j}$ and a corresponding point $\tilde{x}_{j}$ in its universal covering $\tilde{M}_{j}$. The fundamental group $\pi_{1}\left(M_{j}\right)$ acts on $\tilde{M}_{j}$ as deck transformation. Applying [F] Theorem 2.1, [FY1] Proposition 3.6 and [FY2] Theorem 4.1 for our sequence $\left(M_{j}, x_{j}\right)$ and their universal coverings $\left(\tilde{M}_{j}, \tilde{x}_{j}\right)$ and fundamental groups $G_{j}=\pi_{1}\left(M_{j}\right)$, one has

Lemma 4.1. There exist an Alexandrov space $(Y, y)$ and a Lie group $G$ which is a closed subgroup of isometries of $Y$ such that one has $Y / G=X$ and

$$
\lim _{i \rightarrow \infty}\left(\tilde{M}_{i}, G_{i}, \tilde{x}_{i}\right)=(Y, G, y)
$$

Moreover, for any normal subgroup $H$ of $G$ with the properties

(1) $G / H$ is discrete, and 
there exists a sequence of normal subgroups $H_{i}$ of $G_{i}$ such that

(1) $\lim _{i \rightarrow \infty}\left(\tilde{M}_{i}, H_{i}, \tilde{x}_{i}\right)=(Y, H, y)$,

(2) $G_{i} / H_{i}$ is isomorphic to $G / H$ for sufficiently large $i$,

(3) $H_{i}$ is generated by $H_{i}\left(r+\epsilon_{i}\right)$ for some $\epsilon_{i}$ with $\epsilon_{i} \rightarrow 0$.

Next we take the normal subgroup $G_{0}$ of the connected component of the identity element of $G$. Since $G_{0}$ is generated by $G_{0}(\epsilon)$ for any positive number $\epsilon$. We can choose $\epsilon$ to be $\epsilon(n) / 4$ where $\epsilon(n)$ is given by Margulis' Lemma.

Since $G$ is a Lie group, $G / G_{0}$ is discrete. Lemma 4.1 then implies that there exists a sequence of normal subgroups $E_{j}$ of $G_{j}$ such that $G_{j} / E_{j}$ is isomorphic to $G / G_{0}$ for sufficently large $i$. Moreover, $E_{j}$ is generated by $E_{j}(2 \epsilon)$ for large $j$.

From our choice of the number $\epsilon$, Margulis' Lemma implies that the normal subgroup $E_{j}$ is almost nilpotent. Then, Lemma 2.1 yields that $\hat{\mathrm{H}}^{*}\left(M_{j}\right)$ is isometrically isomorphic to $\hat{\mathrm{H}}_{a}^{*}\left(K_{j}\right)$ for a multicomplex $K_{j}$ of $K(\pi, 1)$ type with $\pi=G_{j} / E_{j} \simeq G / G_{0}$.

Since any two $K(\pi, 1)$ multicomplices with isomorphic $\pi$ 's are homotopy equivalent, thus $\hat{\mathrm{H}}^{*}\left(M_{j}\right)$ is isometrically isomorphic to each other for sufficiently large $j$. This leads to a contradiction and Theorem $\mathrm{C}$ follows.

\section{REFERENCES}

[A] M. Anderson, Short geodesics and gravitational instantons, J. Diff. Geom. 31 (1990), $265-275$.

[CC1] J. Cheeger and T. H. Colding, Lower bounds on Ricci curvature and almost rigidity of warped products, Ann. of Math. 144 (1996), 189-237.

[CC2] J. Cheeger and T. H. Colding, On the structure of spaces with Ricci curvature bounded below, I, J. Differ. Geom. 46 (1997), 406-480.

[Br] R. Brooks, Riemann Surfaces and Related Topics, Proceedings of the 1978 Stony Brook Conference, 1980 Princeton University Press, pp. 53-63.

[F] K. Fukaya, Theory of convergence for Riemannian orbifolds, Japan J. Math. 12 (1986), 121-160.

[FY1] K. Fukaya and T. Yamaguchi, The fundamental group of almost nonnegatively curved manifolds, Annals of Math. 136 (1992), 253-333.

[FY2] K. Fukaya and T. Yamaguchi, Isometry group of singular spaces, Math. Zeit. 216 (1994), 31-44.

[G1] M. Gromov, Curvature, diameter and Betti numbers, Comment. Math. Helv. 56 (1981), 179-195.

[G2] M. Gromov, Groups of polynomial growth and expanding maps, Publ. Math. IHES 53 (1981), 53-73.

[G3] M. Gromov, Volume and bounded cohomology, Publ. Math. IHES 56 (1981), 213-307.

[GP] K. Grove and P. Petersen V, Manifolds near the boundary of existence, J. Differ. Geom. 33 (1991), 379-394.

[GLP] M. Gromov, J. Lafontaine and P. Pansu, Structure metrique pour les varites riemanniennes, Cedic / Fernand Nathan, Paris, 1981.

[GPW] K. Grove, P. Petersen and J.-Y. Wu, Geometric finiteness theorems via controlled topology, Invent. Math. 99 (1990), 205-211.

[GT] S. Gallot, Inégalités isopérimétriques, courbure de Ricci et invariants géométriques, I, C. R. Acad. Sci. Sér. I Math. 296 (1983), 333-336.

[HT] J.M. Hirsch and W. Thurston, Foliated bundles, invariant measures and flat manifolds, Ann. of Math. 101 (1975), 369-390.

[I] Ivanov, N. V., Foundations of the theory of bounded cohomology, J. of Soviet Math. 37 (1987), 1090-1114.

[P] P. Petersen V, Gromov-Hausdorff convergence of metric spaces, Proc. Symp. Pure 
[SY] J. Sha and D. Yang, Examples of manifolds of positive Ricci curvature, J. Differ. Geom. 29 (1989), 95-103.

[W1] J.-Y. Wu, Hausdorff convergence and sphere theorems, Proc. Sym. Pure Math. ed. by S.-T. Yau and R. Greene, Vol 54, Part 3 (1993), 685-692.

[W2] J.-Y. Wu, An obstruction to fundamental groups of positively Ricci curved manifolds, Annals of Global Analysis and Geometry 16 (1998), 371-382.

[Y] T. Yamaguchi, Collapsing and pinching under a lower curvature bound, Annals of Math. 133 (1991), 317-357.

Z. SHEN,

Department of Mathematical Sciences, Indiana Univ.-Purdue Univ. at Indianapolis, 402 N. Blackford Street, Indianapolis, IN 46202-3216, U.S.A.

EMAIL: ZSHEN@MATH.IUPUI.EDU

J.-Y. WU,

Department of Mathematics, National Chung Cheng University, Ming-Hsiung, ChiaYI 621, TAIWAN

EMAIL:JYWU@MATH.CCU.EDU.TW 